\title{
Commentary
}

\section{COVID-19: Preparedness in Nuclear Medicine Departments in Singapore and Response to The Global Pandemic}

Pei Ing Ngam, ${ }^{1}$ MBBS, FRCR, MMed, Charles XY Goh, ${ }^{1}$ MBBS, FRCR, MMed, David CE $\underline{\mathrm{Ng}},{ }^{1}{ }_{M B B S,}$ FRCP, FAMS,

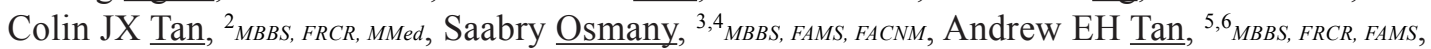

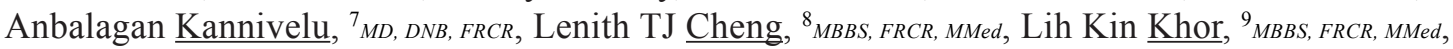
Aaron KT Tong, ${ }^{1} M B B S, M R C P$, FAMS , Kelvin SH Loke, ${ }^{1} M B B S$, MRCP, FAMS, Wai Yin Wong, ${ }^{1} M B B S$, FRCR, FAMS

\section{Introduction}

The first cluster of pneumonia of unknown aetiology patients was reported in Wuhan, China, on 31 December 2019. ${ }^{1}$ The pathogen was subsequently identified as a new strain of coronavirus-severe acute respiratory syndrome coronavirus 2 (SARSCoV-2), which is the causative agent of COVID-19. COVID-19 unfortunately could not be contained in China and the World Health Organization (WHO) declared the outbreak a pandemic on 11 March 2020. ${ }^{2}$

Singapore, with its high population density and status as an international hub for business and tourism, has been badly hit by the outbreak and the economy is expected to shrink between 4 and 7 per cent this year. ${ }^{3}$ The first imported case of COVID-19 was confirmed on 23 January $2020 .^{4}$ The disease continued to spread within the community and Singapore's Ministry of Health $(\mathrm{MOH})$ raised the nation's Disease Outbreak Response System Condition (DORSCON) level from Yellow to Orange 2 weeks later. ${ }^{5}$ On 3 April 2020, the Singapore government announced an elevated set of safe distancing measures, as a "circuit breaker" to limit movements and interactions in public and private places. ${ }^{6}$ As of 3 July 2020, Singapore had reported a total of 44,479 cases of COVID-19 with 26 deaths from COVID-19 complications. ${ }^{7}$

In response to government policies, the key imperatives to strengthen the outbreak management and emergency response system are to treat the suspected and confirmed cases, while avoiding intra-hospital and cross-institutional transmission between staff and patients, and to establish efficient contact tracing. ${ }^{8}$ In this paper, we describe the impact on nuclear medicine services in both public and private sectors in Singapore, focusing on the key factors to consider in terms of processes and policies while being flexible to adapt new practices in this rapidly evolving pandemic.

\section{General Infection Control Measures}

Standard precautions are practised in all public and private hospitals to minimise the risk of infection to patients, family members and healthcare workers as well as controlling COVID-19 transmission.

Current evidence suggests that COVID-19 is transmitted through respiratory droplets and contact routes..$^{9,10}$ Thus, it is mandatory for all nuclear medicine staff to wear surgical masks in clinical areas at all times. Full physical personal protection equipment (PPE), including N95 mask, face shield/ goggles, gowns and gloves, are compulsory when attending to suspected or confirmed cases of COVID-19. Mask fitting and training sessions were organised to assist staff in selecting appropriate mask models and to ensure the proper use of N95 masks.

Social distancing between staff is actively reinforced. All staff are to maintain a minimum distance of 1 metre apart. To facilitate this, staggered meal times and working hours have been implemented to minimise interaction. Shared facilities such as pantries and seating areas now have strict limits on the number of staff allowed at any given time.

Daily self-monitoring for early COVID-19 symptoms is crucial for healthcare workers. ${ }^{11,12}$ Therefore, staff are required to $\log$ their temperature twice a day and are obliged to seek medical attention if they are unwell.

\footnotetext{
${ }^{1}$ Department of Nuclear Medicine and Molecular Imaging, Singapore General Hospital, Singapore.

${ }^{2}$ ParkwayHealth Radiology, Mount Elizabeth Hospital, Singapore.

${ }^{3}$ RadLink PET and Cardiac Imaging Centre, Paragon Medical, Singapore.

${ }^{4}$ Osmany Pte Ltd, Singapore.

${ }^{5}$ Nuclear Medicine Centre, Raffles Hospital, Singapore.

${ }^{6}$ Department of Nuclear Medicine, Farrer Park Hospital, Singapore.

${ }^{7}$ Department of Diagnostic Radiology, Khoo Teck Puat Hospital, Singapore.

${ }^{8}$ Department of Diagnostic Imaging, National University Hospital, Singapore.

${ }^{9}$ Department of Nuclear Medicine, Advanced Medicine Imaging, Singapore.

Address for Correspondence: Dr Ngam Pei Ing, Department of Department of Nuclear Medicine and Molecular Imaging, Singapore General Hospital, Singapore. Email: peiing.ngam@mohh.com.sg
} 


\section{Primary and Secondary Screening}

All patients and visitors undergo primary screening upon entry into the hospital. Individuals are required to fill up a declaration form, providing their personal particulars, travel history, contact history with COVID-19 patients or known clusters of disease, and presence of any flu-like symptoms. Temperature screening systems have been put in place to detect individuals with high body surface temperature. A doctor in-charge is consulted if any patients are found to be symptomatic.

Secondary screening is performed at the departmental level. An isolation room has been designated for any suspected cases that inadvertently arrive at the department. The duty nuclear medicine physician will review the patient in the room with necessary precautions, depending on symptoms and contact history.

Ideally, pre-screening of the patients before their visits by conducting screening questionnaires over the phone a day prior to the appointment/admission is recommended as this can potentially reduce unnecessary patients' trip to the hospital.

According to the WHO's guidelines, a suspected case is defined by clinical presentation and travel/ contact history with COVID-19 patients, while a confirmed case is defined by positive laboratory testing. Although imaging has its role in suspected and confirmed cases, there is no study evaluating the diagnostic accuracy of imaging in asymptomatic contacts of patients with COVID-19. ${ }^{13}$ However, following multiple publications on lung findings on computed tomography (CT) scans in COVID-19 patients, ${ }^{14-16}$ we have implemented screening protocols to pick up incidental suspicious lung findings on the CT component of hybrid positron emission tomography-CT (PET-CT) and single-photon emission computerized tomography-CT (SPECT-CT) scans as well as myocardial perfusion imaging (MPI), where CT attenuation correction has been performed. CT images are screened for new bilateral peripheral ground glass opacities before the patient is allowed to leave the department. If necessary, given the typical midinspiratory $\mathrm{CT}$ acquisition, a dedicated low dose breath-hold CT can be performed to exclude changes secondary to atelectasis. However, considerable overlap in imaging findings leads to diagnostic difficulty in differentiating atypical viral pneumonia from therapyrelated pneumonitis, especially in our practice, which is geared towards the oncology patient population. These patients will first be evaluated by a nuclear medicine physician, and if deemed suspicious after clinical assessment, the on-call infectious disease specialist will be activated and the referring clinician will be alerted. This modified workflow and close communication with our clinician partners allows early detection and isolation of suspicious but asymptomatic patients, and helps to combat spread of COVID-19 in the community.

\section{Segregation of Patients in Space and Time}

Nuclear medicine in Singapore grew out of the Singapore General Hospital's (SGH) Radiotherapy Department (although there was incidental use of scintigraphy in the internal medicine department in SGH) and became an independent 'Department of Nuclear Medicine, SGH' in 1980. We started with Technetium-99m (Tc-99m) radiopharmaceutical based diagnostic imaging and radio-iodine (Iodine-131) therapy for hyperthyroidism and thyroid cancers in 1980s. Up to the mid-1990s, nuclear medicine services provided by SGH, the National Heart Centre Singapore, National University Hospital (NUH) and Mount Elizabeth Hospital sufficiently fulfilled clinical demands. The introduction of clinical F-18fluorodeoxyglucose (F-18 FDG) PET-CT in the early 2000s prompted the setting up of PET-CT services in Singapore with a central cyclotron sited in SGH. Since then, therapeutic nuclear medicine, which was primarily focused on radioiodine treatment of thyroid disease, now encompasses a much broader range of targeted therapies that have entered standard clinical practice. Theranostics, which utilise radioactive tracers in establishing the diagnosis and treatment of cancer, has become increasingly relevant in the era of precision medicine. Singapore is one of the pioneers of theranostics in Southeast Asia and attracts patients from many countries.

The rapid evolution of nuclear medicine has resulted in a remarkable increase in the number of new facilities and resources, at times straining the pre-existing infrastructure of departments already experiencing space constraints. This limitation impedes effective segregation of patients in the event of pandemics. $\mathrm{SGH}$, which has one of the region's largest Nuclear Medicine departments, operates to cater for the demands not only from Singhealth institutions but from other public and private clinics. Perfect segregation of patients is indeed challenging.

Applying the concept of physical segregation during Severe Acute Respiratory Syndrome (SARS) epidemic, ${ }^{11,12}$ we minimise the contact between inpatients and outpatients with the principle of 
segregation in space and time. One of the gamma cameras is designated for all urgent and inpatient cases, while the other two gamma cameras are used for outpatient cases. As there is only a single PETCT scanner and single ultrasound machine, temporal segregation is performed by acquiring all outpatient scans before inpatient cases, with adequate equipment cleaning with alcohol based solution/wipes between cases and compulsory terminal cleaning at the start and end of the day, and after scanning patient with suspected COVID-19 infection. This helps to physically separate these 2 groups of patients. We also space out our appointment slots to minimise patient waiting time and overlap in the department. All doctors, nurses and nuclear medicine technologists attending to patients have their initials documented on the patients' request forms to facilitate contact tracing.

Similar to public hospital practices, isolation rooms are provided for all inpatients and therapy patients in the private hospitals. Provisions need to be made for holding rooms and designated toilets, which are subject to cleaning upon visit completion. The number of attending staff is also kept to a minimum, donning the appropriate PPE. The conduct of patient segregation and isolation precautions exceeds global best practices. Free-standing nuclear medicine centres do not scan inpatients to limit the potential spread of COVID-19, and follow similar screening, isolation, segregation and protection policies tailored to the needs of each individual centre.

Children pose a unique challenge in paediatric nuclear medicine centres. Owing to the necessity of having at least 1 caregiver present all the time, the implementation of distancing or isolation measures is difficult. Furthermore, some paediatric nuclear medicine scans and procedures require multiple visits on separate days (such as for Iodine-131 MIBG scintigraphy). Children who require these procedures must be attended to by team doctors on the same rotation to avoid cross contamination between within and between the departments, as scanning a child usually involves the paediatrician, anaesthesiologist and nuclear medicine physician. For paediatric PET-CT studies, the patient and caregiver are kept isolated in designated uptake rooms to minimise contact with other patients.

\section{Segregation of Workforce}

It is of utmost importance to make sure that our staff remain safe and healthy. Any infected staff member can infect other colleagues or susceptible patients, and potentially lead to a shutdown of the service.
Learning from our painful experiences during SARS in $2003,,^{17,18}$ our staff in public and private practices have been segregated into 2 teams that are meant to complement each other. For instance, the "on-site" team will take charge of the work with potential patients' contact such as running clinic and ward, while the "off-site" team will help with remote reporting of the diagnostic scans. In order to avoid exhaustion of a particular team owing to uneven workload, some hospitals allow the teams to alternate their duties every 2 weeks. This principle of team segregation is widely adopted, including the nuclear medicine division in Khoo Teck Puat Hospital (KTPH), which is staffed with 2 nuclear medicine physicians and 5 radiographers. This ensures continuity of services while maintaining physical segregation to avoid cross-exposure between teams. Remote reporting has also been expanded as needed, to minimise unnecessary travel and exposure.

There is strictly no cross-institution movement for all staff unless approval has been given by $\mathrm{MOH}$ under special circumstances.

\section{Disruptions to the Radioisotope Supply Chain and Provision of Services}

The viral pandemic has led to a disruption in global supply chains across all industries, including the nuclear medicine service. The commonly used radionuclides such as Technetium-99m and Iodine-131 are sourced overseas, and are vulnerable to supply chain disruptions. Although the production of radionuclides remains unaffected, cancellations of international flights during this period have resulted in significant shipment delays. Substantial effort has been put into sourcing alternate supplies and routes of transport by working with procurement departments. All the supplies are bound by contract. There is therefore negligible concern with regard to competitive cost between public and private sectors as this is a nationwide issue and not pertaining to a particular institution. Therefore, a few strategies have been adopted: (1) reschedule less emergent examinations such as bone and thyroid uptake scans; (2) convert the study to a different imaging modality such as echocardiography or CT coronary angiography instead of MPI; and (3) modify the imaging/therapy protocol such as preparing patients awaiting radio-iodine therapy with recombinant TSH (Thyrogen) instead of thyroxine withdrawal, owing to the possibility of unexpected treatment delays resulting in prolonged hypothyroidism.

In order to minimise the risk of occupational exposure, aerosol-generating procedures such as ventilation scans 
for pulmonary embolism diagnosis have been suspended. MPI is now routinely carried out using pharmacologic stress, and exercise stress testing is avoided in most circumstances. A 1-day rest-pharmacologic stress protocol is preferred to the routine 2-day stress-rest protocol, as this minimises the number of visits to the department.

Oncologic therapies such as radio-iodine (Iodine-131) therapy, Lutetium-177 peptide receptor radionuclide therapy (PRRT), Lutetium-177 prostate specific membrane antigen (PSMA), and Yttrium-90 radioembolisation therapies, are deemed essential services and will continue for all local patients. Within public healthcare institutions, foreign patients have been advised to seek treatment in their home countries to minimise imported cases. Private nuclear medicine facilities cater for the clinical needs of many foreign patients, although their numbers have significantly reduced as a result of travel restrictions. The provision of nuclear medicine services in the private facilities adhere to governmental restrictions on non-essential medical services. A specific concern for this sector is the heightened patient risk profiling and screening when travel restrictions are lifted in the future.

\section{Integration of electronic platforms}

Technological advances act as an integration platform for doctors, nurses and allied health professionals to facilitate multidisciplinary patient care.

With the implementation of strict institutional, inter- and intra-departmental segregation, electronic applications such as TigerConnect, ${ }^{19} \mathrm{Zoom}^{20}$ and Webex ${ }^{21}$ are utilised as to enable remote participation in multidisciplinary tumour board teleconferences, department or administrative meetings which provides continuity of these collaborative activities while maintaining safe distancing. Tele-reporting allows flexibility of cross coverage, which increases productivity and reduces the accumulation of stress as a result of limited manpower. These platforms are crucial in hospitals without an in-house nuclear medicine physician. Diagnostic nuclear medicine studies performed in other institutions are tele-reported in SGH. Yttrium-90 radioembolisation therapy in Tan Tock Seng Hospital (TTSH) is remotely co-managed with the nuclear medicine department in KPTH. ${ }^{22}$ Patient's data and liver-lung shunt images are transferred securely, and discussions on feasibility and therapy planning are made through electronic platforms. On the day of Yttrium-90 delivery, audiovisual teleconferencing allows for collaborative communication between the KPTH nuclear medicine physician and the TTSH interventional radiologist. To date, 15 Yttrium-90 radioembolisation therapies have been successfully conducted this way. In the private hospitals, mirror reporting stations using cloud based tele-radiology platforms for image and report transmission are used for tele-reporting.

In addition, these applications are used for peer review learning (PRL) sessions and didactic residents' teaching in public hospitals. Our residents are given the opportunity to present their scholarly activities at local and international conferences. Unfortunately, many major conferences including our locally organised SGH Nuclear Medicine Update (NMU) have been cancelled or postponed as a proactive measure in preventing community spread of the novel coronavirus. A major annual nuclear medicine meeting usually hosted in North America by the Society of Nuclear Medicine and Molecular Imaging will be entirely virtual this year. As specialists, faculty and residents become more comfortable with learning via electronic platforms, we envisage that these platforms will continue to be used to complement conventional teaching methods in providing a holistic learning experience to our profession.

These electronic applications are not flawless. Recently, there have been rising concerns on the security of patient data shared over these platforms. Adequate education on the use of inbuilt security features is needed for all users and administrators to minimise risk.

\section{Conclusion}

Our past experiences dealing with the 2003 SARS coronavirus and $2009 \mathrm{H} 1 \mathrm{~N} 1$ helped prepare us to face this new pandemic. The rapid and coordinated response of the nuclear medicine community to the COVID-19 pandemic has been instrumental in minimising disruption to essential nuclear medicine services, so we can be there for our patients in this crisis. As we move forward in this exigent situation, we remain vigilant in screening and detecting possible COVID-19 cases in our centres, to mitigate exposure risk to our staff and provide continuing care for our patients.

\section{REFERENCES}

1. Zhu N, Zhang D, Wang W, Li X, Yang B, Song J, et al. A Novel Coronavirus from Patients with Pneumonia in China, 2019. The New England journal of medicine. 2020;382(8):727-33.

2. World Health Organization. WHO Director-General's opening remarks at the media briefing on COVID-19 (11 March 2020). 
Available at: https://www.who.int/dg/speeches/detail/who-directorgeneral-s-opening-remarks-at-the-media-briefing-on-covid-19---11march-2020. Accessed on 3 July 2020.

3. The Straits Times. Singapore heads for worst recession since independence; economy to shrink by $7 \%$ to $4 \%$ on Covid- 19 impact. 26 May 2020. Available at: https://www.straitstimes.com/business/ economy/spore-to-sink-into-deeper-recession-than-expected-2020growth-forecast-cut-to. Accessed on 3 July 2020.

4. Ministry of Health Singapore. Confirmed imported case of novel coronavirus infection in Singapore; multi-ministry taskforce ramps up precautionary measures. 23 January 2020. Available at: https:// www.moh.gov.sg/news-highlights/details/confirmed-imported-caseof-novel-coronavirus-infection-in-singapore-multi-ministry-taskforceramps-up-precautionary-measures. Accessed on 3 July 2020.

5. Ministry of Health Singapore. Risk assessment raised to DORSCON orange. 7 February 2020 Available at: https://www.moh.gov. sg/news-highlights/details/risk-assessment-raised-to-dorscon-orange. Accessed on 3 July 2020.

6. Ministry of Health Singapore. Circuit breaker to minimise further spread of COVID-19.2020 [Available from: https://www.moh. gov.sg/news-highlights/details/circuit-breaker-to-minimise-furtherspread-of-covid-19. 3 April 2020. Accessed on 3 July 2020.

7. The Straits Times. 169 new Covid-19 patients in Singapore, including 11 in the community. 3 July 2020. Available at: https:// www.straitstimes.com/singapore/169-new-covid-19-patients-insingapore-including-11-in-the-community-3-imported. Accessed on 3 July 2020

8. Lam WW, Loke KS, Wong WY, Ng DC. Facing a disruptive threat: how can a nuclear medicine service be prepared for the coronavirus outbreak 2020? European journal of nuclear medicine and molecular imaging. 2020;47(7):1645-8.

9. Li Q, Guan X, Wu P, Wang X, Zhou L, Tong Y, et al. Early Transmission Dynamics in Wuhan, China, of Novel CoronavirusInfected Pneumonia. The New England journal of medicine. 2020;382(13):1199-207.

10. Huang C, Wang Y, Li X, Ren L, Zhao J, Hu Y, et al. Clinical features of patients infected with 2019 novel coronavirus in Wuhan, China. Lancet (London, England). 2020;395(10223):497-506.

11. Tan CC. SARS in Singapore--key lessons from an epidemic. Annals of the Academy of Medicine, Singapore. 2006;35(5):345-9.
12. Goh KT, Cutter J, Heng BH, Ma S, Koh BK, Kwok C, et al. Epidemiology and control of SARS in Singapore. Annals of the Academy of Medicine, Singapore. 2006;35(5):301-16.

13. World Health Organization. Use of chest imaging in COVID-19 - World Health Organization. 11 June 2020. Available at: https://www.who. int/publications/i/item/use-of-chest-imaging-in-covid-19. Accessed on 3 July 2020.

14. Ai T, Yang Z, Hou H, Zhan C, Chen C, Lv W, et al. Correlation of Chest CT and RT-PCR Testing in Coronavirus Disease 2019 (COVID-19) in China:AReport of 1014 Cases. Radiology. 2020:200642.

15. Qin C, Liu F, Yen TC, Lan X. (18)F-FDG PET/CT findings of COVID-19: a series of four highly suspected cases. European journal of nuclear medicine and molecular imaging. 2020; 47(5):1281-6.

16. Xu X, Yu C, Qu J, Zhang L, Jiang S, Huang D, et al. Imaging and clinical features of patients with 2019 novel coronavirus SARS-CoV-2. European journal of nuclear medicine and molecular imaging. 2020;47(5):1275-80.

17. Tsou IY, Goh JS, Kaw GJ, Chee TS. Severe acute respiratory syndrome: management and reconfiguration of a radiology department in an infectious disease situation. Radiology. 2003; 229(1):21-6.

18. Chen M, Leo YS, Ang B, Heng BH, Choo P. The outbreak of SARS at Tan Tock Seng Hospital--relating epidemiology to control. Annals of the Academy of Medicine, Singapore. 2006; 35(5):317-25.

19. Andrew Brooks MD BB, Jeffrey Evans. TigerConnect: TigerConnect, Inc.; 2010 Available at: https://tigerconnect.com/. Accessed on 3 July 2020.

20. Yuan E. Zoom: Zoom Video Communications, Inc.; 2011 [Available at: https://zoom.us/. Accessed on 3 July 2020.

21. Subrah Iyar MZ. Webex Meeting: Cisco Webex; 1995 [Available at: https://www.webex.com/video-conferencing. Accessed on 3 July 2020.

22. Quek LNA, Kannivelu A, Pua U. Yttrium-90 Radioembolization: Telemedicine during COVID-19 outbreak, opportunity for prime time. Journal of nuclear medicine : official publication, Society of Nuclear Medicine. 2020. 